\title{
A coupled performance and thermal model for radio-frequency gridded ion thrusters ${ }^{\star}$
}

\author{
Mantas Dobkevicius ${ }^{\mathrm{a}}$ and Davar Feili \\ University of Southampton, Southampton, Hampshire, SO17 1BJ, UK \\ Received 16 April 2016 / Received in final form 25 July 2016 \\ Published online 1 November 2016 \\ (c) The Author(s) 2016. This article is published with open access at Springerlink.com
}

\begin{abstract}
Recently proposed space missions such as Darwin, eLISA and NGGM have encouraged the development of electric propulsion thrusters capable of operating in the micro-Newton $(\mu \mathrm{N})$ thrust range. To meet these requirements, radio frequency $(\mathrm{RF})$ gridded-ion thrusters need to be scaled down to a few centimeters in size. Due to the small size of these thrusters, it is important to accurately determine the thermal and performance parameters. To achieve this, a multi-physics performance model has been developed, composed of plasma discharge, 2D axisymmetric ion extraction, 3D electromagnetic and RF circuit models. The plasma discharge model itself is represented using 0D global, 2D axisymmetric and 3D molecular neutral gas, and Boltzmann electron transport sub-models. A 3D thermal model is introduced to determine the temperature distribution for various throttle points, using as inputs the plasma and electromagnetic field heating values obtained from the performance model. This also allows the validation of the performance model itself. Additionally, we analyze the effect the thruster's temperatures play on the plasma properties/performance and vice versa. The model is based on the RIT 3.5 thruster developed for the NGGM mission geometry and predicts the RIT 3.5 experimental data within approximately $10 \%$.
\end{abstract}

\section{Introduction}

In 1884, Hittorf proposed the idea of plasma generation using radio-frequency (RF) fields [1]. In 70's Loeb [2] was one of the first to apply the idea of using RF plasmas for electrostatic space propulsion. Following an Ariane-5 upper stage malfunction in 2001, a RITA (Radio-Frequency Ion Thruster Assembly) system was operated for around one year as the main means of propulsion in order to shift ESA's ARTEMIS spacecraft to the desired mission orbit [3]. This proved the RF thrusters' versatility and reliability. One of the main advantages of RF thrusters is that they can sustain an electrodeless discharge and are easily throttleable by varying coil power. This is because the ion production and acceleration mechanisms are separate, which allows RF thrusters to be scaled down. Therefore, with missions such as Darwin [4], eLISA [5] and NGGM [6] requiring thrusters working in the $\mu \mathrm{N}$ range and with high throttling capabilities, RF thrusters have been considered as one of the main candidates in fulfilling the mission requirements. However, working of an RF thruster involves many different physics phenomena as shown in Figure 1, making a holistic approach to the performance analysis difficult. Furthermore, due to the small size of these thrusters and due to high frequencies, plasma measurements are challenging to perform. Additionally, in

\footnotetext{
* Contribution to the Topical Issue "Physics of Ion Beam Sources", edited by Holger Kersten and Horst Neumann.

a e-mail: md4g09@soton.ac.uk
}

order to make the scaled-down thrusters applicable to the aforementioned space missions, they must consume low amounts of power, have a high efficiency, and must be prevented from overheating.

The main physical processes and components that are needed to fully describe an RF ion thruster are shown in Figure 1 . The plasma is created by accelerating electrons with an azimuthal electric field $E_{\theta}$ due to a magnetic field $B$ which is produced by passing a current $I_{c}$ through a coil wrapped around an insulating gas chamber. As a result, a neutral gas at a density $n$ is ionized to produce a plasma with a density $n^{+}$. The ions are extracted and focused with electrically biased multi-aperture grids. The extraction grids also play a role in controlling the neutral gas pressure inside the chamber since the propellant that is not ionized leaks through the grids. The power to the coil is provided by an RF circuit composed of a Radio-Frequency Generator (RFG) and a matching network. An ideal thruster would transform all input power into thrust. However, a substantial amount of power is lost from the plasma to the discharge chamber walls or radiated to the environment. Furthermore, there are losses associated with the electromagnetic eddy current production in the thruster's components. All these contribute to the heating of the thruster - requiring a thermal analysis. Therefore, in order to have a complete model of the thruster, thermal effects have to be consistently included. This is because the thruster's temperatures affect the plasma properties through the neutral gas pressure 


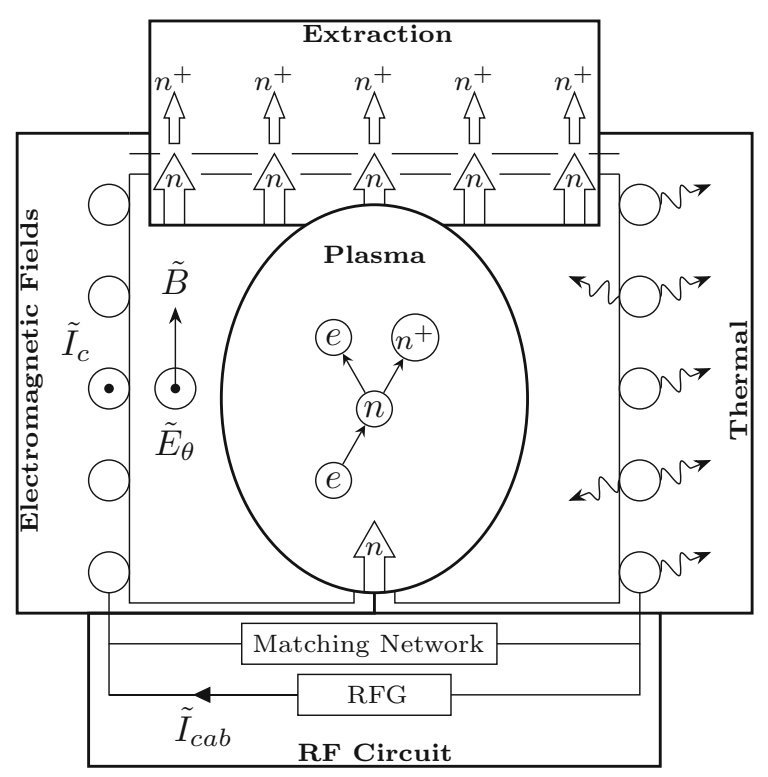

Fig. 1. Schematics highlighting the main RF gridded ion thruster components, RF circuit and physical processes occurring in the plasma.

and eddy current losses through a change in the component electrical conductivity. In turn, the plasma properties affect the thruster's temperatures as well. Thus, the RF thruster operation encompasses many different areas of physics, requiring a self-consistent holistic modeling approach in order to accurately predict the performance.

To understand the RF thruster behavior, we first need to model the coupling between the coil and plasma. Thompson [7] was one of the first to model the RF plasma discharge to be a secondary of an air-core transformer. His approach is still commonly used because it does not require the explicit knowledge of the spatial field and plasma distributions. Using Thompson's approach, Piejak et al. [8] managed to describe the RF discharges through the use of electrical circuit parameters such as the RF current, voltage and power. The main RF discharge modeling efforts and assumptions are summarized in references $[9,10]$. To simulate an actual RF thruster, Goebel developed an analytical volume-averaged (0D) plasma model [11]. This allowed him to calculate the plasma power losses for a desired beam current. Nevertheless, he did not look at the electromagnetic aspect of RF thruster operation, which prevented him from calculating the total thruster input power and efficiency. Chabert et al. [12] extended Goebel's model and included the electromagnetic part by introducing the aforementioned transformer assumptions. Volkmar et al. developed a 3D Biot-Savart solver to calculate the coil impedance and used the magnetic diffusion equation to determine the electromagnetic field distribution inside the plasma represented by the uniform 0D model [13]. The model was aimed to act as a tool for designing RF thrusters by giving a complete description of the thruster. Nevertheless, the model suffered from a few shortcomings. First, it excluded the thruster's components from the electromagnetic field analysis, which affects the power balance and matching conditions. Sec- ond, the plasma conductivity was assumed to be uniform. However, the plasma conductivity varies as a function of the discharge chamber radius. This variation greatly influences the power that is transferred from the coil to plasma. Additionally, the ion extraction model was based on the space-charge limited Child-Langmuir's law, without explicitly modeling the plasma extraction. Finally, the thruster's temperatures were not simulated at all, and the temperature effect on the thruster's performance was not accounted for.

To the authors' knowledge, there is no self-consistent thermal model that is capable of accounting for plasma and RF heating and, in turn, for thruster's temperature effect on plasma properties and RF heating. Full RF thruster thermal models are particularly challenging to construct because the magnitude of RF heating (via eddy currents) is dependent on the thruster's component temperatures due to a change in their electrical conductivity. Gartner et al. [14] did create a thermal model of a RIT 2.5 thruster. However, their model only included the RF heating contribution. The plasma heating was not modeled because they did not develop a plasma model to account for different boundary heat fluxes. Creating such a plasma model introduces a great deal of complexity, since many plasma parameters are unknown or cannot be measured. Therefore, their model was only applicable for conditions when there is no plasma and the thruster is heated solely by the eddy currents. Nevertheless, a few thermal models have been developed for ring cusp gridded ion thrusters such as the one by Van Noord described in reference [15]. The main goal of the present paper is to introduce a full RF thruster model that could be used to predict the thruster's performance and temperature distribution in a consistent fashion with solution times of less than an hour, acting as a fast, efficient and accurate thruster design tool.

In this paper we look at all five major branches of $\mathrm{RF}$ thruster operation: electromagnetic field generation, plasma production, ion extraction, RF circuit analysis and temperature distribution. A schematic RF thruster model including all five branches is shown in Figure 1. The electromagnetic model uses a full 3D geometry of the thruster including the ion optics and case in order to solve electromagnetic field equations in COMSOL [16]. The plasma itself is represented using a $0 \mathrm{D}$ sub-model similar to the ones developed in references [17-19]. However, to increase the accuracy of the model, the ion density is assumed to vary radially. Electrons are defined using the electron energy distribution function obtained by solving a highfrequency two-term approximation Boltzmann equation in COMSOL. The neutral gas pressure and density inside the plasma are determined using a sub-model that solves molecular flow equations in COMSOL. The ion extraction model is obtained by modifying an open-source software called IBSIMU developed by Kalvas [20]. The RF circuit model is implemented in PSpice software [21]. The thermal model is based on the full 3D thruster geometry and uses the heating values from the plasma and electromagnetic models to determine the complete temperature distribution. The results from the thermal model are then fed back 
to the plasma and electromagnetic models until a consistent solution is reached. Finally, the model is benchmarked against the experimental results of RIT 3.5 miniature RF ion thruster designed for the NGGM mission [6].

\section{Plasma discharge model}

In this section we describe a plasma discharge model that is used to calculate volume-averaged plasma parameters. Additionally, the plasma discharge model is needed to compute the heat fluxes from the plasma to the thruster in order to determine the temperature distribution. Using the plasma discharge model we also analyze the effect the chamber wall temperature plays on the neutral gas pressure and, thus, plasma properties.

\subsection{Neutral gas}

The neutral gas density $n_{0}$ in the discharge chamber defines the magnitude of the electron temperature and the collision frequency between electrons and neutrals. In determining the neutral gas density, we first calculate the ion optics Clausing factor $\eta_{c}$ using a 2D axisymmetric ion optics geometry in a molecular flow sub-module developed in COMSOL. Then we use a 3D molecular flow sub-model of the discharge chamber by taking the previously calculated Clausing factor, the discharge chamber wall temperature $T_{w}$ and the neutral gas mass flow rate $\dot{m}$ as inputs. The Clausing factor is used as a boundary condition on the screen grid apertures in the $3 \mathrm{D}$ sub-model that reflects a fraction $R$ of incident molecules $G$ back to the discharge chamber expressed as:

$$
R=\left(1-\eta_{c} f_{s o}\right) G,
$$

where we model the screen grid apertures as a surface with a mathematical open area fraction $f_{\text {so }}$.

The discharge chamber wall temperature is determined from the thermal model that will be described in Section 4 . The input neutral gas mass flow rate to the model $\dot{m}$ is calculated as the mass flow rate of the neutral gas that leaks through the grids $\dot{m}_{\text {out }}$ and is not part of the ion beam. This is done by subtracting the equivalent mass flow rate required to obtain a specific ion beam current $I_{b}$ from the total input mass flow rate $\dot{m}_{i n}$

$$
\dot{m}_{\text {out }}=\dot{m}_{\text {in }}-I_{b} g_{0},
$$

where $g_{0}$ is the conversion factor from Amperes to sccm equal to $13.938 \mathrm{sccm} / \mathrm{A}$. We obtain the beam current $I_{b}$ using an ion extraction model developed in IBSIMU [20]. In this model we determine the ion optics focusing efficiency parameter $T_{\text {eff }}$, which is influenced by the meniscus shape and hence the upstream plasma properties, i.e. the electron temperature $T_{e}$ and plasma sheath edge density $n_{s}$. Therefore, we define the extracted ion current as $I_{b}=T_{\text {eff }} I_{B}$, where $I_{B}$ is the total Bohm current flowing towards the screen grid area $A_{s}$

$$
I_{B}=n_{s} u_{B} A_{s} e
$$

where $e$ is the electron charge, $u_{B}=\sqrt{e T_{e} / M_{i}}$ is the Bohm (ion) velocity and $M_{i}$ is the ion mass.

\subsection{Plasma properties}

The plasma parameters are calculated using a 0D submodel. In determining the rate coefficients $\gamma_{k}$ for each collision process $k$, we obtain an electron energy distribution function (EEDF) $F_{0}$ by solving a two-term approximation Boltzmann equation for the oscillating fields using a submodel in COMSOL. As main inputs, the model takes the ionization degree $\beta=n_{i} /\left(n_{i}+n_{0}\right)$, the reduced angular frequency $\omega / n_{0}$ and the neutral gas temperature $T_{0}$, which is assumed equal to the discharge chamber wall temperature $T_{w}$. The collision cross-sections $\rho_{k}$ for each collision process $k$ are provided as well [22]. We then derive the effective electron-neutral collision frequency $\nu_{\text {eff }}$ from a high-frequency electron mobility $\mu$

$$
\mu=\frac{e / m}{\nu_{e f f}+j \omega_{e f f}},
$$

where $\omega_{\text {eff }}$ is the effective angular frequency that, for the RIT 3.5 thruster, we set equal to the applied frequency $\omega$ [10], and $m$ is electron mass. Since the RIT 3.5 thruster works at a low pressure of around 1 mTorr we also include collisional heating $[23,24]$ processes whereby we express the plasma skin depth as [19]:

$$
\delta_{p}^{-1}=\frac{\omega}{c} \operatorname{Im}\left[\epsilon_{p}^{1 / 2}\right]
$$

where $\epsilon_{p}$ is the relative plasma dielectric constant

$$
\epsilon_{p}=1-\frac{\omega_{p e}^{2}}{\omega\left(\omega-j \nu_{t o t}\right)},
$$

with the plasma frequency given by $\omega_{p e}=\sqrt{n_{i} e^{2} / m \epsilon_{0}}$, where $c$ is the speed of light and $\epsilon_{0}$ is the permeability of free space. To represent the stochastic heating mechanism, Lieberman and Lichtenberg have introduced an equivalent stochastic frequency $\nu_{\text {stoc }}$ using the skin depth $\delta_{p}$ and the average electron thermal velocity $\bar{v}_{e}[19]$

$$
\nu_{\text {stoc }} \approx \frac{\overline{v_{e}}}{4 \delta_{p}} .
$$

Neglecting the electron-ion collision frequency $\nu_{e i}$, the total collision frequency $\nu_{t o t}$ can be determined as the sum of the effective $\nu_{\text {eff }}$ and stochastic $\nu_{\text {stoc }}$ collision frequencies, i.e.:

$$
\nu_{t o t}=\nu_{e f f}+\nu_{s t o c} .
$$

The final $\nu_{\text {tot }}$ value is obtained by iteratively solving equations (5)-(8) until convergence is achieved. Using the Boltzmann sub-model we are able of obtaining the fractional power losses $f_{k}$ for each collision process $k$. We can express the power lost in ionizing the gas as [25]:

$$
P_{i}=\gamma_{i} N_{1} N_{2} V U_{i} e,
$$


where $V$ is the discharge chamber volume, $U_{i}$ is the ionization potential, which for Xenon is equal to $12.13 \mathrm{eV}$, $N_{1}$ and $N_{2}$ are the two species densities, $\gamma_{i}$ is the ionization rate coefficient. As a result, the total collisional power expanded in creating a plasma is $P_{\text {coll }}=P_{i} / f_{i}$, where $f_{i}$ is the fraction of total power expanded to ionization. Similarly, the power losses for other collision processes are given as $P_{k}=f_{k} P_{\text {coll }}$. This allows the powers lost to ionization, excitation and elastic collision processes to be determined.

Furthermore, we improve on the uniform plasma density assumption by introducing a radial variation in the plasma density through the $h$ parameter equal to

$$
h=\frac{n_{i}(r)}{n_{i}(0)},
$$

where $n_{i}(0)$ is the ion density at the center of the discharge (from now on referred to as simply $n_{i}$ ) and $n_{i}(r)$ is the ion density at a location $r$ from the center. This fraction has been shown to be largely dependent on the neutral gas density $n_{0}$ through the ion mean free path $\lambda_{i}$ equal to $\lambda_{i}=1 /\left(n_{0} \rho_{s}\right)$, with $\rho_{s}$ being the total ion scattering cross-section. Neglecting the ion diffusion terms, the ion density variation in the radial direction $h_{r}$ is $[17,19]$ :

$$
h_{r} \approx \frac{0.8}{\left[4+\frac{R}{\lambda_{i}}\right]^{1 / 2}}
$$

while the ion density variation in the axial direction $h_{l}$ is:

$$
h_{l} \approx \frac{0.86}{\left[3+\frac{l}{2 \lambda_{i}}\right]^{1 / 2}} .
$$

Based on experimental [26] and analytical [17,19] observations, we assume that the ion density distribution follows a circular profile that varies from 1 at the centre of the discharge chamber $(r=0)$ to the $h_{r}$ value at the radial boundary $(r=R)$ as given by the equation

$$
h=\sqrt{1-\left(\sqrt{1-h_{r}^{2}} \frac{r}{R}\right)^{2}} .
$$

Since $\gamma_{i}\left(T_{e}\right)$, the electron temperature is found by setting the rate of ion production (left side of Eq. (14)) equal to the rate of ion loss (right side of Eq. (14)). In deriving the equation, it is assumed that ions are lost by flowing to the chamber wall $A_{c}$ and screen wall $A_{s}$ areas with the Bohm velocity $u_{B}$ where, if not extracted, they recombine to produce a neutral atom. The final particle balance equation is:

$$
n_{0} \gamma_{i}\left(T_{e}\right) V=u_{B}\left(h_{r} A_{c}+h_{l} A_{s}\right) .
$$

Note that the electron temperature does not depend on the ion density $n_{i}$ since it cancels out from the above equation. The plasma potential is obtained by assuming that the electron and ion fluxes to the boundaries are ambipolar (or equal). Therefore, the plasma potential is given by a well-known floating potential equation

$$
\phi=T_{e} \ln \sqrt{M_{i} /(2 m \pi)} .
$$

\subsection{Heat fluxes}

In order to account for the plasma's effect on the thruster heating, the particle currents within the discharge chamber are determined. Using the conservation of particles and energy $[17,19]$, and the ambi-polar flux assumption, the total ion or electron current flowing towards the discharge chamber wall $A_{c}$ is:

$$
I_{w i}=h_{r} n_{i} u_{B} A_{c} e .
$$

As discussed in Section 2. 1, the beam current is:

$$
I_{b}=T_{e f f} h_{l} n_{i} u_{B} A_{s} e \Gamma_{b},
$$

where $\Gamma_{b}$ is the flatness of the beam used to define the radial variation in the $h_{l}$ parameter. If we take the RIT 3.5 ion optics system as an example, we see that the beam flatness $\Gamma_{b}$ is around $85 \%$ since the grid apertures are made only in a high-density plasma region to give a flat beam. The ion current to the screen grid area $A_{s}$ is equal to the remaining current that is not extracted through the grids to form the ion beam

$$
I_{s i}=\left(1-T_{e f f}\right) h_{l} n_{i} u_{B} A_{s} e \Gamma_{b} .
$$

Assuming ambipolar losses again, the electron current to the screen grid is $I_{s e}=I_{b}+I_{s i}$.

As previously defined, there are losses associated with a production of the plasma itself. One of the losses is the collisional power loss $P_{\text {coll }}$ made of ionization $P_{i}$, excitation $P_{x}$ and elastic scattering $P_{\text {scat }}$ losses. However, a substantial power loss is additionally incurred due to various particle fluxes as indicated in Figure 2. One particle loss mechanism occurs when, upon reaching the wall, these particles are accelerated by the electrostatic fields in the plasma and then deposit their energy to the wall, causing it to heat up. Additionally, the heat can also be transferred by the excited neutral particles that radiate energy to the thruster's boundaries or out of the discharge chamber into the beam. We determine the heating to a specific boundary from the excited neutral flux based on its surface area with respect to the total surface area $A_{w}$ as seen by the plasma [15].

The discharge chamber wall heating by ions and electrons is:

$$
P_{c}=I_{w i}\left(\phi+\frac{5}{2} T_{e}+U_{i}\right),
$$

whereas, the heating from excited neutrals is:

$$
P_{x c}=\frac{A_{c}}{A_{w}} P_{x} .
$$

The screen grid heating due to electrons is:

$$
P_{s e}=I_{s e}\left(w_{s}+2 T_{e}\right),
$$

where $w_{s}$ is the screen grid work function. The screen grid heating due to ions is:

$$
P_{s i}=I_{s i}\left(\phi+\frac{1}{2} T_{e}+U_{i}-w_{s}\right) .
$$




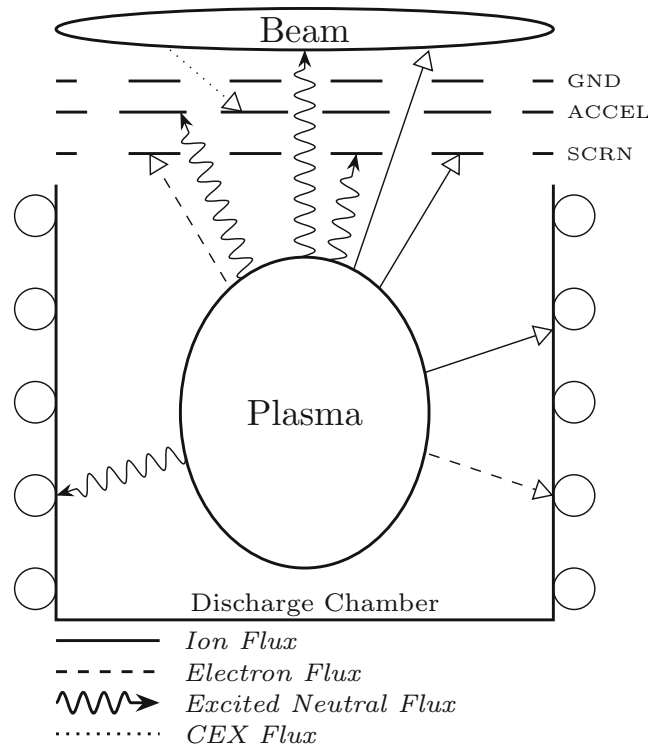

Fig. 2. Particle fluxes in a radio-frequency gridded ion thruster plasma.

Finally, the screen grid heating due to excited neutrals is:

$$
P_{x s}=\frac{\left(1-f_{o s}\right) A_{s}}{A_{w}} P_{x}
$$

where $f_{o s}$ and $f_{o a}$ represent the screen and accel grid open area fractions, respectively. The heating of the accel grid comes mainly from the excited neutral radiation given by:

$$
P_{x a}=\frac{\left(1-f_{o a}\right) f_{o s} A_{s}}{A_{w}} P_{x},
$$

and charge exchange (CEX) ions

$$
P_{C E X}=I_{c e x}\left(U^{-}+U_{i}-w_{a}\right),
$$

where $I_{\text {cex }}$ is the CEX particle current, usually less than $2 \%$ of the beam current, and $w_{a}$ is the accel grid work function. The power processed by the RFG power supply also includes the excited neutral radiation power that escapes through the grid system to the beam

$$
P_{\text {xout }}=\frac{f_{o a} f_{o s} A_{s}}{A_{w}} P_{x},
$$

and the power that escapes with the ions in the beam

$$
P_{b}=I_{b}\left(\phi+\frac{1}{2} T_{e}+U_{i}\right) .
$$

The total discharge power $P_{d}$ that is needed to generate a required beam current plasma at a specified mass flow rate is a sum of all the aforementioned losses, i.e.:

$P_{d}=P_{s c a t}+P_{c}+P_{x c}+P_{s e}+P_{s i}+P_{x s}+P_{x a}+P_{x o u t}+P_{b}$

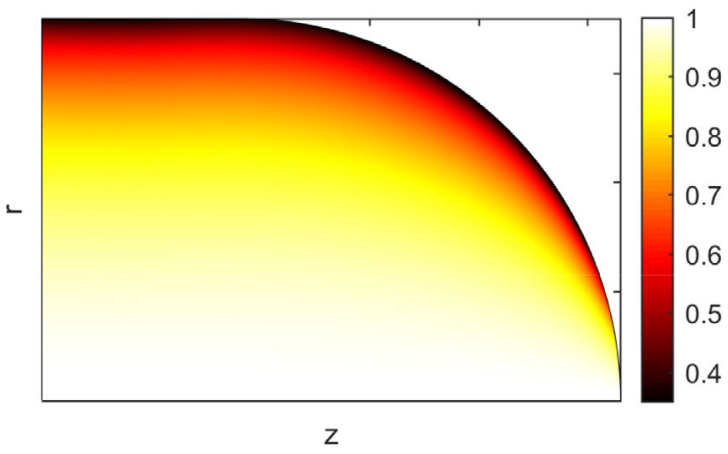

Fig. 3. Ion density distribution (or the $h$ parameter) inside the RIT 3.5 thruster discharge chamber normalized by a center plasma density.

\section{Electromagnetic and RF circuit models}

A 3D electromagnetic model has been created in COMSOL in order to solve Maxwell's equations in the plasma and thruster. The model treats the plasma as a simple solid with a complex electrical conductivity given as [27]:

$$
\sigma_{p}=\frac{n_{i} e^{2}}{m\left(\nu_{t o t}+i \omega_{e f f}\right)},
$$

where the plasma parameters are obtained from the plasma discharge model. Representing the plasma conductivity as $\sigma_{p}=\sigma_{r}-\sigma_{i} j$ introduces a phase lag between the electric field and current through the imaginary part in order to simulate the finite electron inertia [17]. Since the ion density varies radially, we represent the plasma electrical conductivity as $\sigma_{p} \propto h n_{i}$ using the $h$ factor defined in equation (13) and depicted in Figure 3.

Using the plasma electrical conductivity we can calculate the power that is lost in the plasma due to induced electromagnetic fields $E$ as:

$$
W_{p}=\frac{1}{2} \iiint \Re\left(\sigma_{p} \cdot|E|^{2}\right) \mathrm{d} V,
$$

the same equation can be used to find the power lost in the thruster's components as well by replacing the $\sigma_{p}$ value with the component $k$ electrical conductivity $\sigma_{k}$. However, we can also calculate the power lost in the thruster's component/plasma using Ohm's law as:

$$
W_{k}=\frac{1}{2} R_{k}^{\prime} I_{c}^{2}
$$

where $R_{k}^{\prime}$ is the reflected component/plasma resistance, or the resistance as seen by the RFG, and $I_{c}$ is the coil current. The $R_{k}^{\prime}$ parameter depends solely on the component/plasma conductivity, geometry and relative distribution with respect to the coil (or $E$ field strength) as seen in equation (30). Therefore, based on the electrical conductivity, for each reflected resistance value $R_{k}^{\prime}$ we can determine the power lost in this component depending on the applied coil current $I_{c}$. 


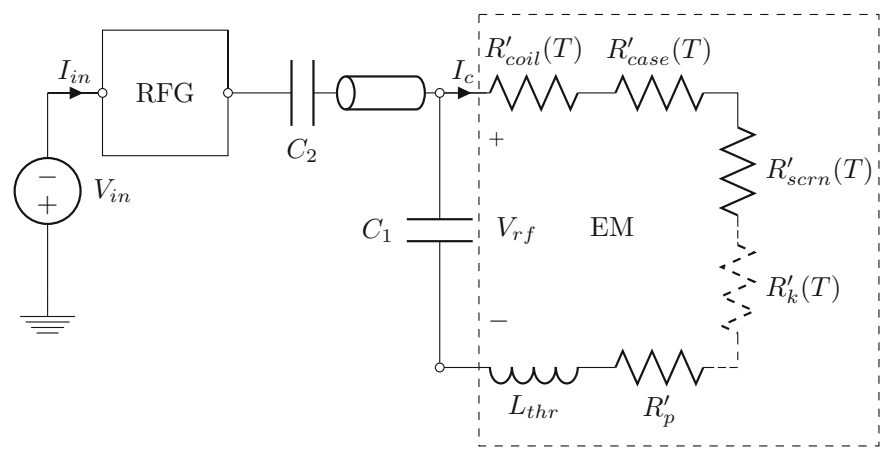

Fig. 4. Equivalent RF ion thruster representation in terms of electrical components.

We can transform the thruster to an equivalent $\mathrm{RF}$ circuit through Maxwell's equations as illustrated in Figure 4 . As can be seen the circuit is composed of a number of reflected resistances: the plasma resistance $R_{p}^{\prime}$, coil resistance $R_{c o i l}^{\prime}$, screen grid resistance $R_{s c r n}^{\prime}$ and thruster case resistance $R_{\text {case }}^{\prime}$. There are many additional resistances denoted by $R_{k}^{\prime}$ which come from such components as electrodes, bolts, screws, nuts, etc. There is also a total equivalent thruster inductance $L_{t h r}$ used to describe the inductive component of the thruster behavior. Additionally, it is important to mention that the thruster's component reflected resistances are functions of the temperature $R_{k}^{\prime}(T)$ because the electrical conductivity $\sigma_{k}(T)$ is a function of the temperature. Figure 5 shows how the reflected resistances vary with temperature for the thruster case, screen grid and coil.

The circuit depicted in Figure 4 also includes an RF generator (RFG), a matching network composed of capacitors $C_{1}$ and $C_{2}$ (normally part of the RFG) and a coaxial cable in order to accurately represent the thruster's behavior. The whole circuit is modeled in PSpice in a similar manner as shown in Figure 4. The PSpice model gets the knowledge about the plasma and thruster from the electromagnetic model in terms of parameters that are indicated in Figure 4 as "EM" and outlined with a dashed box. In modeling the circuit, we first obtain the reflected plasma resistance $R_{p}^{\prime}$ based on the electrical plasma conductivity $\sigma_{p}$ that arises due to a specific beam current $I_{b}$ that is extracted. Then we obtain all other reflected resistances. We model the circuit by changing the input current to the RFG $I_{\text {in }}$ until we reach a required coil current $I_{c}$. The coil current is determined for given plasma conditions as:

$$
I_{c}=\sqrt{\frac{2 P_{d}}{R_{p}^{\prime}}} .
$$

In deriving this equation we assume that in steady state conditions the power lost in the plasma due to electromagnetic fields $W_{p}$ must be equal to the discharge power loss $P_{d}$. We also use the concept of reflected resistance. Once all the resistances and the total inductance are known, we can determine the input power that needs to be supplied to the thruster, the total thruster efficiency, RFG input voltage $V_{i n}$, power lost in the coaxial cable/RFG and so on.

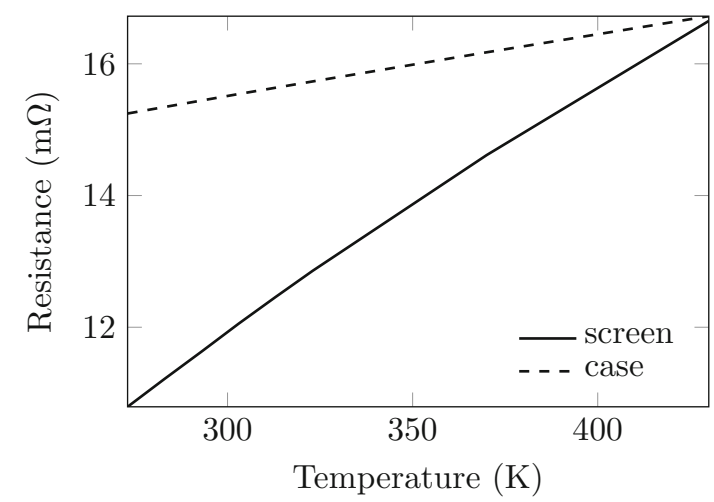

(a)

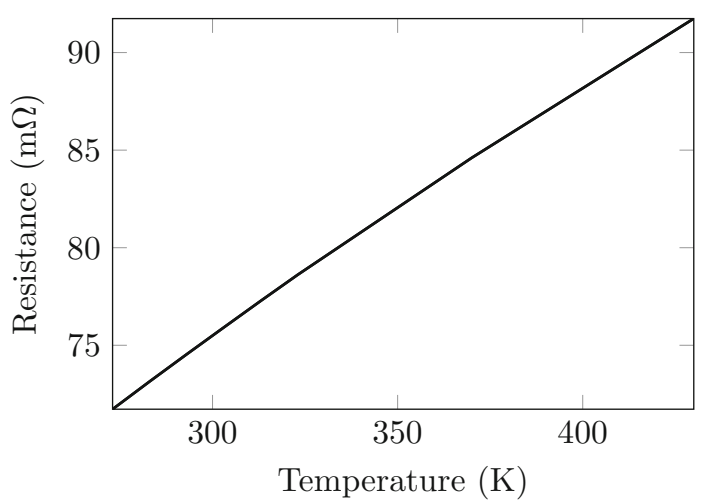

(b)

Fig. 5. (a) Screen and case, (b) coil reflected resistance variation with temperature.

\section{Thermal model}

A thermal model based on the RIT 3.5 thruster geometry has been designed in order find the thruster's surface temperature distribution. The model is used to observe what effect the thruster's temperatures have on the electromagnetic heating losses in the conductors, as well as on the plasma properties. Additionally, the model is needed to analyze and help solve any potential thermal issues present in the thruster such as thermal stresses, ion optics and coil thermal expansions, etc. It is also very important to make sure that the matching network capacitors do not overheat. To find the temperature distribution, a 3D thermal model was developed in COMSOL by solving a steady state heat equation

$$
\Delta(-\kappa \Delta T)=Q_{i}
$$

where $Q_{i}$ represents the heat sources within the system and $\kappa$ is the thermal conductivity. There are two heat sources, neglecting the contribution from the environment: plasma fluxes and RF heating. The plasma heating $P_{k}$ due to charged particle fluxes and excited neutral radiation was described in Section 2.3, whereas the RF heating due to eddy currents in the thruster components $W_{k}$ was defined in Section 3. 


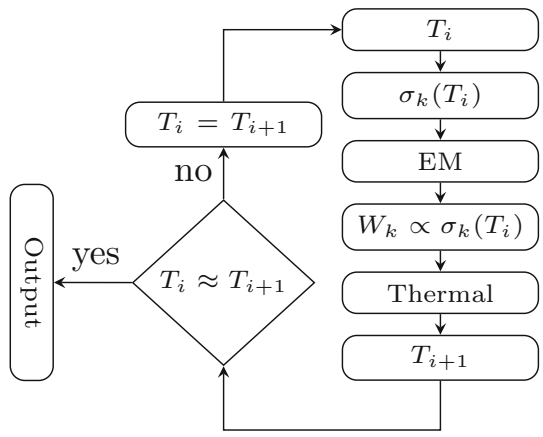

(a)

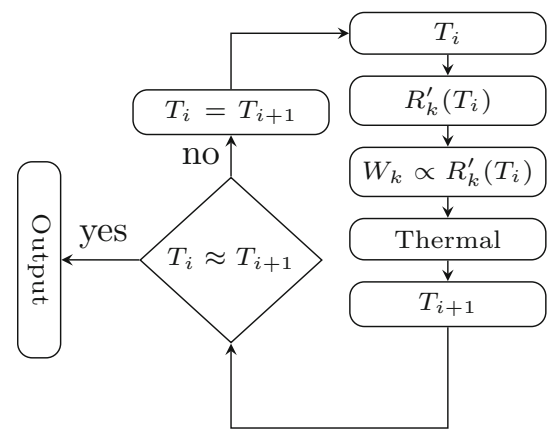

(b)

Fig. 6. Flow chart depicting (a) full and (b) reduced coupling between the thruster's temperatures and the RF heating.

It is assumed that the thruster loses heat to the vacuum chamber either by conductance through a contact point with the thruster's attachment plate, or by surface radiation. The thruster is modeled as being attached to an infinitely large body at a set temperature $T_{a m b}$, which is used to mimic the vacuum chamber. To complete the heat balance equations, surface-to-surface and ambient radiation mechanism are included as:

$$
Q_{\text {rad }}=\epsilon\left(G_{\text {rad }}-k_{B} T^{4}\right),
$$

where $\epsilon$ is the emissivity of the material which is set equal to the absorptivity $\alpha$ across the full wavelength spectrum due the gray-body assumption [28] and $k_{B}$ is the Boltzmann constant. The $G_{r a d}$ parameter represents the incoming heat flux composed of the ambient $G_{a m b}$ and mutual heat fluxes $G_{m}$ between the components

$$
G_{r a d}=G_{m}+G_{a m b} .
$$

In order to save computational time, radiation groups are defined. We define a radiation group as a collection or a set of components, including the environment, that have considerably large view factors with respect to each other [28]. Therefore, we solve the radiation equations only within a particular radiation group.

Our investigations have shown that the contact resistances between components are very important in accurately predicting the temperature distribution. However, these are hard to estimate due to many unknown parameters. Nevertheless, the contact resistance values were obtained using the data available in literature and trial and error methods. We represent the contact resistance $U_{c}$ between two adjacent component surfaces using the Mikic's elastic model [29]

$$
U_{c}=\frac{1.54 \kappa_{c} m_{a s p}}{\tau_{a s p}}\left(\frac{\sqrt{2} p_{c}}{m_{a s p} E_{c}}\right)^{0.94},
$$

where $m_{a s p}$ and $\tau_{a s p}$ are the effective root-mean-square (RMS) contact surface asperities' slope and roughness, respectively, $p_{c}$ is the contact pressure and $\kappa_{c}$ is the average conductivity of the surfaces with thermal conductivities $\kappa_{1}$ and $\kappa_{2}$ in contact [29]

$$
\kappa_{c}=\frac{2 \kappa_{1} \kappa_{2}}{\kappa_{1}+\kappa_{2}} .
$$

The equivalent contact elastic modulus $E_{c}$ is calculated using the elastic modulus $E_{1}$ and $E_{2}$, as well as Poisson's ratios $\nu_{1}$ and $\nu_{2}$ of the surfaces 1 and 2 in contact [29]

$$
\frac{1}{E_{c}}=\frac{1-\nu_{1}^{2}}{E_{1}}+\frac{1-\nu_{2}^{2}}{E_{2}}
$$

Note that the material emissivities $\epsilon$ and thermal conductivities $\kappa$ were taken from the data sheets provided by the manufacturers of the thruster's components. The thermal conductivities were set as functions of temperature $\kappa(T)$ to get a more accurate temperature distribution.

Figure 6a shows the solution method for a fully coupled thermal system where RF heating is the dominant mechanism of heat generation. First, note that the thruster's temperatures affect the component electrical conductivity $\sigma_{k}$ and, as a result, the RF heating magnitude $W_{k}$ changes. When we set the new heating values into the thermal model, we get new temperature values. We have to repeat this process until the temperatures become self-consistent with the RF heating magnitude. It was observed that it is very hard to obtain convergence using such a method because of a high degree of coupling. Therefore, when solving for the RF heating it was decided to use a solution method depicted in Figure 6b. Here, instead of calculating the RF heating based on the change in the component's electrical conductivity by using the electromagnetic model (EM), we represent all components through reflected resistances $R_{k}^{\prime}$ that are pre-calculated in advance and plotted versus temperature as was shown in Figure 5. This helps to achieve the solution much faster and in a more stable fashion.

In creating the thermal model as few modifications as possible to the original thruster geometry were made. This meant that there were tens of components and hundreds of contact surfaces to mesh and apply boundary conditions to. However, it proved to be very computationally expensive to keep the original ion optics geometry as shown in Figure 7a. This was mainly due to a high number of apertures that needed to be meshed and resolved in solving the radiative heat transfer equations. An accurate representation of the heat radiation through the ion optics is very important in the overall heat balance. This is because the accel grid apertures are smaller than the screen grid 


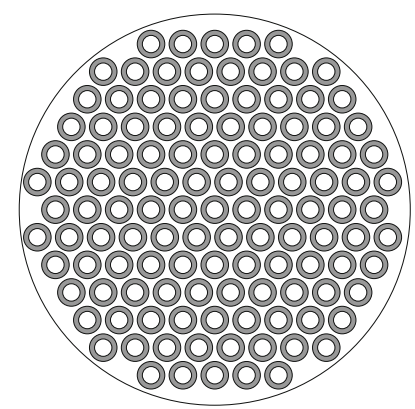

(a)

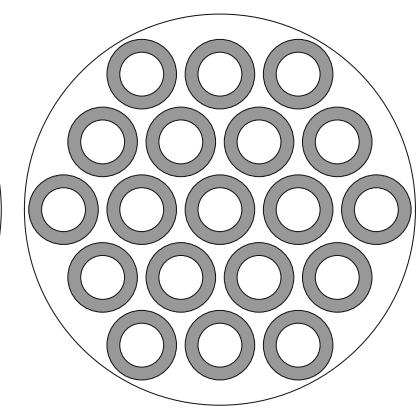

(b)
Fig. 7. (a) Original and (b) modified RIT 3.5 ion optics geometry. White + Gray $=$ Scrn, White $=$ Accel, Gray $=$ Scrn Accel.

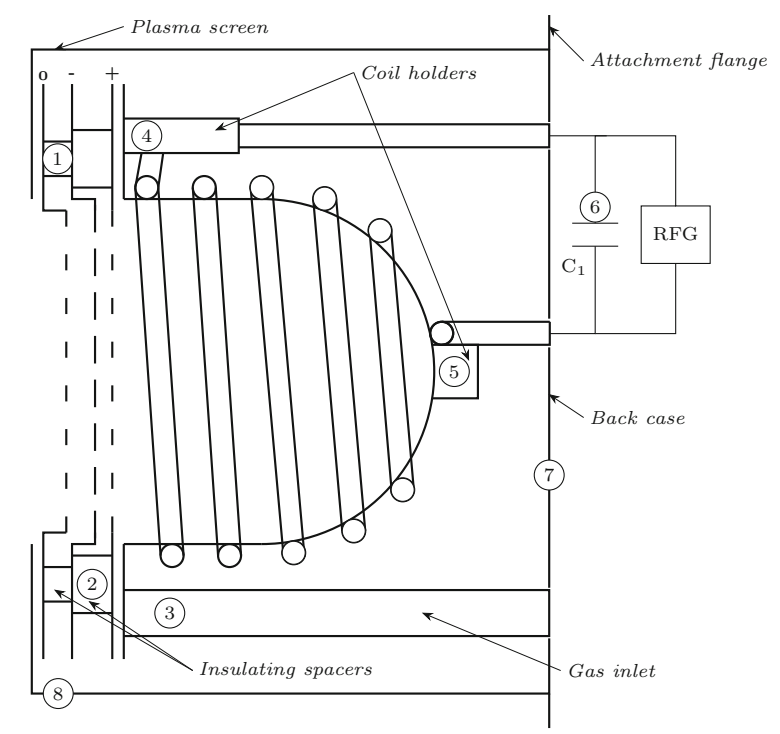

Fig. 8. A schematic RIT 3.5 thruster geometry and thermocouple locations.

apertures and, therefore, some of the heat radiated by the chamber (and the screen grid itself) to the environment is blocked, which greatly influences the overall heat balance. As a result, this heats up the accel grid as well as the rest of the thruster's components. The grid system was simplified as shown in Figure $7 \mathrm{~b}$ by reducing the number of apertures to just 19. However, the accel grid open area (white area) and the screen grid area blocked by the accel grid (gray area) were kept the same. This was done to keep the same radiative heat transfer balance as in the original grid design. Our studies have shown that, by using such a method, there is only a minor effect in terms of the overall thruster temperature distribution.

Figure 8 depicts a schematic RIT 3.5 geometry and the locations of 8 temperature sensors used to validate the model against the experimental temperature measurements. We used PT100 four-wire temperature sensors that were inserted into a ceramic housing and glued to the locations depicted in Figure 8. The sensors were connected to a Keithley data acquisition device that was calibrated to read the changes in the resistance, allowing the temper-

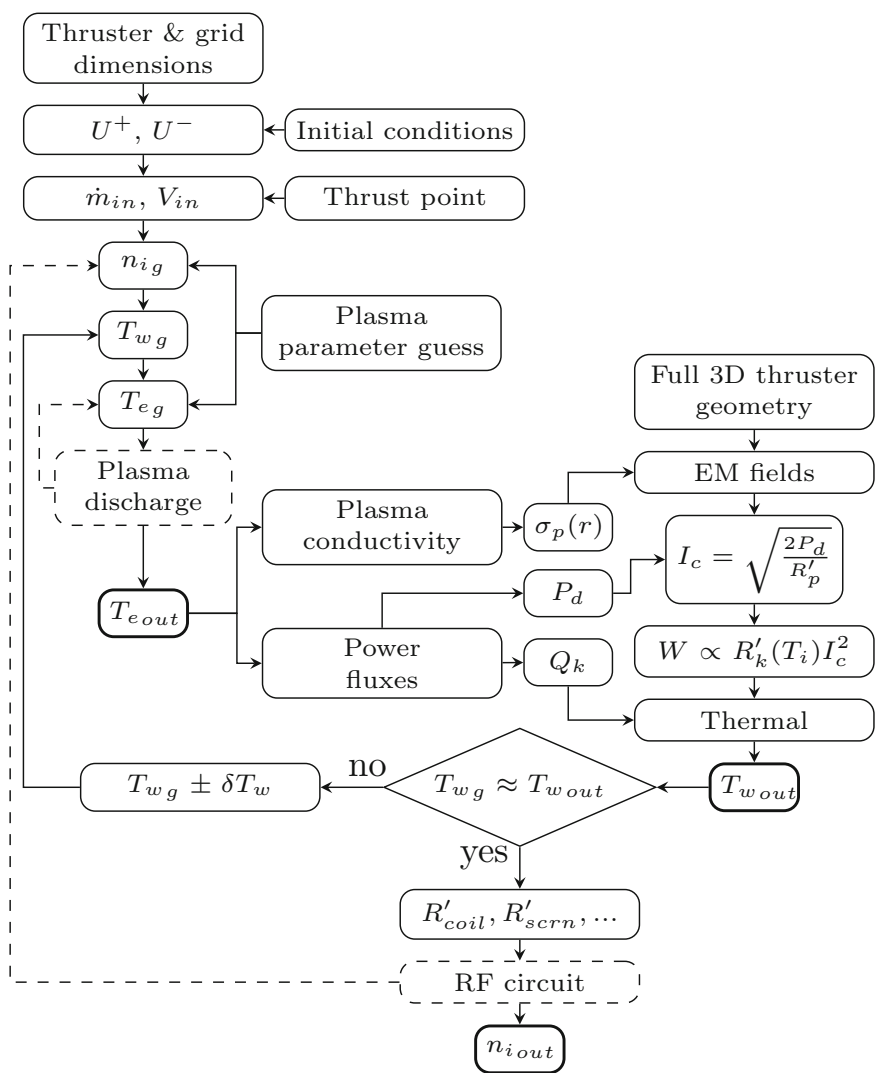

Fig. 9. Main model solution method flow chart. Dashed lines indicate an iterative solution method.

ature values to be extrapolated. We were particularly concerned with the T6 temperature sensor which was measuring the matching capacitor's temperature, since it should not go above $200{ }^{\circ} \mathrm{C}$. The figure also indicates the main components that are present in the thruster. A particular emphasis should be placed on the attachment flange because its contact with the vacuum chamber determines the contact resistance and greatly influences the temperature distribution. There were also plastic insulating holders used to secure the coil in place. The ion optics grids were separated with ceramic spacers.

\section{Solution method}

A schematic of the complete performance and thermal model is shown in Figure 9. The main model consists of different models and sub-models defined in the previous sections that are coupled together through Matlab to easily control the input variables and output parameters for a self-consistent solution. First, the thruster and grid dimensions are given to the model, together with the screen grid voltage $U^{+}$(from now on referred to as beam voltage $V_{b}$ ) and accel grid voltage $U^{-}$. Then, as was performed in the RIT 3.5 experimental campaign, the desired input mass flow rate $\dot{m}_{i n}$ is set together with the RFG voltage $V_{i n}$ as a thrust point. The system is solved for three unknowns: the ion density $n_{i}$, chamber wall temperature $T_{w}$ 
and electron temperature $T_{e}$. Therefore, at the beginning of each iteration, guesses to these unknown parameters are provided. As a result, there are three iteration branches; the first solves for the electron temperature $T_{e}$, the second solves for the chamber wall temperature $T_{w}$ and the final one solves for the RFG voltage $V_{i n}$. For simplicity and clarity, we denote the plasma discharge branch that solves for the electron temperature $T_{e}$ using dashed lines. In this branch we compare the guessed electron temperature to the output electron temperature. If the two do not match, we change the guess values in an iterative manner until a solution is obtained. The same method is applied in solving the RF circuit branch for the RFG input voltage $V_{i n}$.

All the input/output information is passed to the models in a consecutive order as indicated by the arrow directions. The output from each model is used as an input for the next one and so on. At the end of the first iteration branch, we obtain a value for the electron temperature $T_{\text {eout }}$. Using this, we calculate the plasma electrical conductivity and the power fluxes from the plasma. Calculating the plasma power fluxes gives the total power needed to sustain the plasma discharge $P_{d}$ and the individual fluxes to thruster's surfaces or the environment $Q_{k}$. We then use the calculated plasma electrical conductivity $\sigma_{p}(r)$ and the $P_{d}$ values in the electromagnetic model to calculate the required coil current $I_{c}$ in order to sustain the discharge. Having obtained the coil current, we calculate (through Ohm's law) the RF heating values $W_{k}$. Finally, the heat fluxes and the RF heating values are used to represent the heat sources in the thermal model. From the thermal model, we obtain the temperature distribution. Especially, we are interested in the chamber wall temperature $T_{w o u t}$. If the guessed chamber wall temperature $T_{w g}$ does not match the output temperature $T_{\text {wout }}$, we change the $T_{w g}$ value and iterate again. Once a self-consistent temperature distribution is obtained, we determine all the thruster's component reflected resistances and use them in the RF circuit PSPice model to determine the RFG input voltage $V_{i n}$. If this voltage does not match the input voltage $V_{i n}$ that was used in defining the thrust point, the ion density guess value $n_{i g}$ is changed in incremental steps. The iterations in both branches are performed using Matlab's built-in global optimization functions. Once we reach a consistent solution in all three branches, we stop the iterations and output the results.

\section{Results and discussion}

In this section, we present the main results obtained from the study and give a brief discussion of the most important findings. First, we perform a validation of the performance/plasma model. A plot of how the RFG input power varies with the mass utilization efficiency given as $\eta_{m}=\left(I_{b} g_{0}\right) / \dot{m}_{\text {in }}$ is shown in Figure 10 . Such plots are commonly used to characterise the gridded ion thruster performance. As can be seen, the model predicts the RFG input power with a high accuracy, with a maximum error of around $10 \%$.

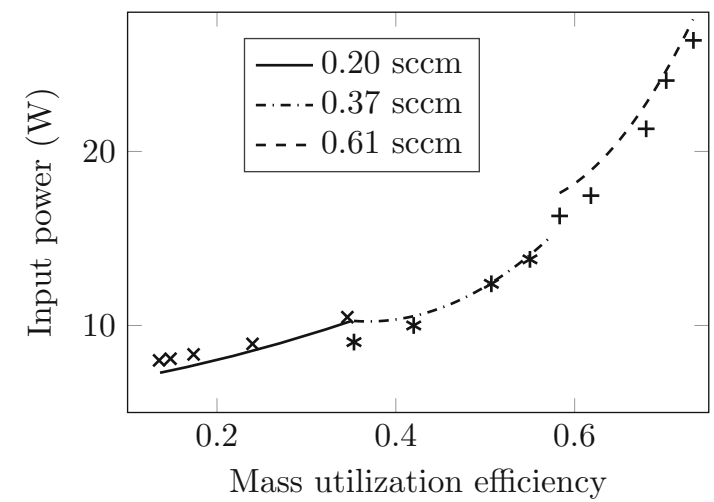

Fig. 10. RFG input power variation with the mass utilization efficiency for $0.2 \mathrm{sccm}, 0.37 \mathrm{sccm}$ and $0.61 \mathrm{sccm}$ propellant mass flow rates compared with the experimental data $\mathrm{x},{ }^{*},+$, respectively.

Table 1. RF heating magnitudes for different RFG input power values without plasma.

\begin{tabular}{cccccccc}
\hline$P_{R F G}(\mathrm{~W})$ & 18.0 & 16.0 & 14.0 & 12.0 & 10.0 & 8.0 & 7.0 \\
$W_{\text {coil }}(\mathrm{W})$ & 9.5 & 8.5 & 7.4 & 6.4 & 5.4 & 4.3 & 3.7 \\
$W_{\text {scrn }}(\mathrm{W})$ & 2.3 & 2.0 & 1.7 & 1.5 & 1.3 & 1.0 & 0.9 \\
$W_{\text {case }}(\mathrm{W})$ & 0.35 & 0.3 & 0.26 & 0.22 & 0.19 & 0.15 & 0.13 \\
$P_{\text {thr }}(\mathrm{W})$ & 12.2 & 10.8 & 9.4 & 8.1 & 6.9 & 5.45 & 4.7 \\
$\eta_{w}(\%)$ & 68.0 & 68.0 & 67.0 & 68.0 & 69.0 & 68.0 & 67.0 \\
\hline
\end{tabular}

To validate the thermal model, we first simulate the thruster's temperatures without the plasma present, i.e. only with the RF eddy current heating as seen in Table 1. This allows us to check that the thermal resistances, thermal conductances, surface emissivities, boundary conditions, etc. are correct, without the possibility of having an incorrect plasma model. As observed in the table, a majority of the RF heating power (around $77 \%$ ) goes to the coil, while approximately $20 \%$ is lost to the screen and $3 \%$ to the case. It can also be seen that only around $68 \%$ of the total RFG input power $P_{R F G}$ reaches the thruster $P_{t h r}$ as given by the power transfer efficiency $\eta_{w}$. This is regardless of the input power, since when there is no plasma, the total thruster resistance stays about constant. The rest of the input power is lost due to Ohmic losses inside the coaxial cable and the RFG itself.

Having obtained the RF heating values, we perform thermal simulations in order to determine the TS1 to TS8 sensor temperatures. In Table 2 we compare the modeled sensor temperatures for different RFG input powers against experimental data without plasma. From the table we can see that the agreement between the experimental results and the model is good, the largest discrepancies are observed in the temperatures measured on the $C_{1}$ capacitor by the sensor TS6, reaching up to $10 \%$. To better understand the overall temperature distribution and to check the accuracy of the model, we also construct a 3D surface temperature plot as shown in Figure 11.

Based on the percentage deviations in both the predicted thruster performance parameters and temperatures, we assume that the model is accurate enough 
Table 2. Thruster's temperature distribution for various RFG input powers without plasma.

\begin{tabular}{|c|c|c|c|c|c|c|c|c|c|c|c|c|c|c|}
\hline \multirow[t]{2}{*}{$P_{R F G}(\mathrm{~W})$} & \multicolumn{2}{|c|}{18.0} & \multicolumn{2}{|c|}{16.0} & \multicolumn{2}{|c|}{14.0} & \multicolumn{2}{|c|}{12.0} & \multicolumn{2}{|c|}{10.0} & \multicolumn{2}{|c|}{8.0} & \multicolumn{2}{|c|}{7.0} \\
\hline & Mod. & Exp. & Mod. & Exp. & Mod. & Exp. & Mod. & Exp. & Mod. & Exp. & Mod. & Exp. & Mod. & Exp. \\
\hline$T_{1}\left({ }^{\circ} \mathrm{C}\right)$ & 66 & 71 & 58 & 66 & 53 & 60 & 50 & 55 & 46 & 49 & 40 & 43 & 39 & 41 \\
\hline$T_{2}\left({ }^{\circ} \mathrm{C}\right)$ & 71 & 74 & 62 & 69 & 58 & 63 & 54 & 56 & 48 & 50 & 42 & 45 & 40 & 42 \\
\hline$T_{3}\left({ }^{\circ} \mathrm{C}\right)$ & 59 & 65 & 54 & 61 & 51 & 56 & 45 & 50 & 42 & 45 & 37 & 41 & 33 & 38 \\
\hline$T_{4}\left({ }^{\circ} \mathrm{C}\right)$ & 58 & 58 & 67 & 54 & 45 & 49 & 46 & 45 & 42 & 40 & 38 & 36 & 36 & 34 \\
\hline$T_{5}\left({ }^{\circ} \mathrm{C}\right)$ & 79 & 80 & 71 & 74 & 60 & 67 & 58 & 60 & 51 & 53 & 45 & 47 & 41 & 44 \\
\hline$T_{6}\left({ }^{\circ} \mathrm{C}\right)$ & 106 & 113 & 94 & 104 & 83 & 93 & 76 & 83 & 68 & 72 & 57 & 62 & 51 & 57 \\
\hline$T_{7}\left({ }^{\circ} \mathrm{C}\right)$ & 38 & 42 & 43 & 40 & 40 & 37 & 32 & 34 & 30 & 31 & 28 & 30 & 28 & 29 \\
\hline$T_{8}\left({ }^{\circ} \mathrm{C}\right)$ & 43 & 41 & 38 & 39 & 36 & 36 & 36 & 34 & 33 & 31 & 30 & 29 & 30 & 28 \\
\hline
\end{tabular}

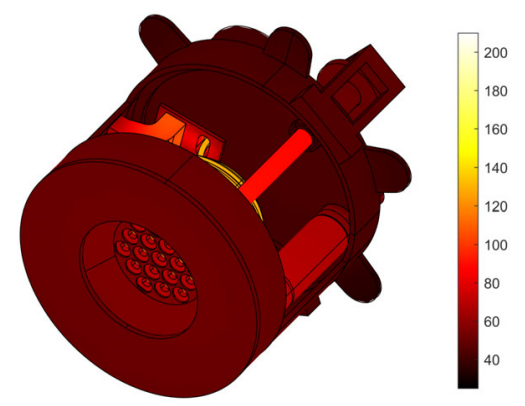

Fig. 11. RIT 3.5 thruster surface temperature distribution for an RFG input power of $18 \mathrm{~W}$ without plasma.

to perform thermal simulations with the plasma present. First, we estimate the RF and plasma heating values for different throttle points based on the beam voltage $V_{b}$ and extracted beam current $I_{b}$ as shown in Table 3 . The table indicates the total power absorbed by plasma $Q_{\text {tot }}$ and how much of this power is lost to the chamber walls $Q_{c}$, screen $Q_{\text {scrn }}$, accel grid $Q_{\text {accel }}$ and beam $Q_{\text {beam }}$. Similarly, the table shows the total power loss due to eddy current RF heating $W_{\text {tot }}$ and how much of this power is lost to the screen $W_{\text {scrn }}$, coil $W_{\text {coil }}$ and case $W_{\text {case }}$. Combining these power losses, we estimate how much power is lost in the thruster altogether $P_{t h r}$. Then by adding losses in the RFG itself and the coaxial cable, we estimate the total RFG input power to the thruster $P_{R F G}$, allowing us to calculate the power transfer efficiency $\eta_{w}$.

It can be observed that when the plasma is present, the power transfer efficiency is much higher compared to the case without the plasma. This is because the presence of plasma dramatically increases the total thruster resistance since there is an additional reflected plasma resistance $R_{p}^{\prime}$ component. Furthermore, the power transfer efficiency goes up as the RFG input power increases, reaching $87 \%$ at around $30 \mathrm{~W}$. This happens mainly due to a rise in the plasma density and electron temperature which increase the reflected plasma resistance. As we go down in power, we also go down in plasma density and, therefore, the power transfer efficiency decreases to $68 \%$, which is the same as in the case where there is no plasma at all, as was discussed previously based on the results from Table 1. Therefore, as far as the RFG operation is concerned, a low-density plasma acts just like the vacuum.

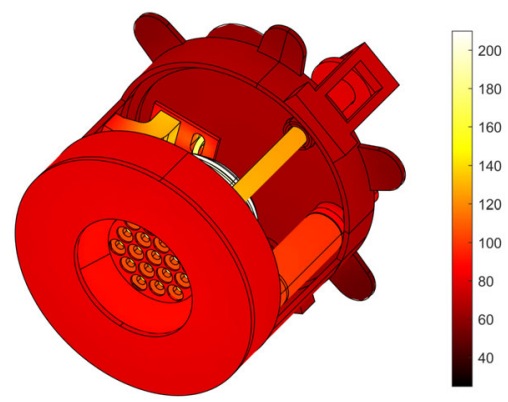

Fig. 12. RIT 3.5 thruster surface temperature distribution for the $V_{b}=1625 \mathrm{~V}$ and $I_{b}=38 \mathrm{~mA}$ throttle point.

In Table 4 we give the temperature distribution based on the aforementioned throttle points. If we take the first throttle point given as $V_{b}=1625 \mathrm{~V}$ and $I_{b}=38 \mathrm{~mA}$, we see that the discrepancy between the modeled and measured temperatures for the TS6 sensor is still the same $6 \%$ as without the plasma. This is very important not only because it confirms that our thermal model is correct, but also that the plasma model is correct as well. If the plasma model was incorrect, we would get temperatures that do not correspond to the measured ones due to wrong plasma heating values. Therefore, the thermal model can also be used to check the accuracy of the plasma model. By analyzing the rest of the table data it can be observed that the maximum error does not go above $14 \%$, with the majority of predicted temperature values falling within $10 \%$ of the measurements. A 3D surface temperature distribution for the aforementioned throttle point is shown in Figure 12. As the plot indicates, the temperatures are much higher than in the case without plasma. For instance, the coil temperature reaches more than $200{ }^{\circ} \mathrm{C}$, which is an increase of about $50 \%$. This makes the coil the hottest component in the thruster. The matching capacitors reach about $140{ }^{\circ} \mathrm{C}$, which is within their operational range. The screen and accel grids are at about $120^{\circ} \mathrm{C}$.

To better understand how the thruster's temperatures affect the plasma properties and performance, we take a case where we set the input mass flow rate $\dot{m}_{i n}$ as a constant and vary the RFG input power. Varying the input power changes the ion density and, in turn, alters the mass utilization efficiency $\eta_{m}$ through the beam current $I_{b}$ as defined in the equation $\eta_{m}=\left(I_{b} g_{0}\right) / \dot{m}_{i n}$. Figure 13 shows how the coil $T_{c}$, chamber wall $T_{w}$, screen grid $T_{s c r n}$ 
Table 3. Plasma $Q$ and RF heating $W$ magnitudes for different throttle points based on the beam voltage $V_{b}$ and beam current $I_{b}$.

\begin{tabular}{cccccccc}
\hline$V_{b}(\mathrm{~V})$ & 1625 & 1500 & 1350 & 1000 & 1200 & 1000 & 262 \\
$I_{b}(\mathrm{~mA})$ & 38.0 & 31.6 & 26.6 & 19.3 & 13.2 & 4.8 & 1.85 \\
$P_{R F G}(\mathrm{~W})$ & 29.7 & 29.4 & 28.5 & 22.1 & 15.0 & 9.4 & 7.5 \\
$Q_{c}(\mathrm{~W})$ & 14.2 & 13.2 & 12.2 & 8.5 & 4.9 & 1.9 & 1.0 \\
$Q_{\text {scrn }}(\mathrm{W})$ & 3.2 & 3.1 & 2.9 & 1.95 & 1.1 & 0.42 & 0.26 \\
$Q_{\text {accel }}(\mathrm{W})$ & 0.47 & 0.35 & 0.3 & 0.22 & 0.14 & 0.05 & 0.03 \\
$Q_{\text {beam }}(\mathrm{W})$ & 1.54 & 1.43 & 1.25 & 0.95 & 0.59 & 0.2 & 0.07 \\
$Q_{\text {tot }}(\mathrm{W})$ & 19.4 & 18.1 & 16.6 & 11.7 & 6.7 & 2.5 & 1.4 \\
$W_{\text {coil }}(\mathrm{W})$ & 5.0 & 5.4 & 5.6 & 4.1 & 3.9 & 3.2 & 2.9 \\
$W_{\text {scrn }}(\mathrm{W})$ & 1.2 & 1.3 & 1.35 & 1.1 & 0.9 & 0.77 & 0.69 \\
$W_{\text {case }}(\mathrm{W})$ & 0.16 & 0.17 & 0.17 & 0.14 & 0.12 & 0.1 & 0.09 \\
$W_{\text {tot }}(\mathrm{W})$ & 6.36 & 6.87 & 7.12 & 5.22 & 4.92 & 4.07 & 3.68 \\
$P_{\text {thr }}(\mathrm{W})$ & 25.7 & 24.9 & 23.7 & 16.9 & 11.7 & 6.6 & 5.1 \\
$\eta_{w}(\%)$ & 87.0 & 85.0 & 83.0 & 77.0 & 78.0 & 70.0 & 68.0 \\
\hline
\end{tabular}

Table 4. Thruster's temperature distribution for different throttle points.

\begin{tabular}{|c|c|c|c|c|c|c|c|c|c|c|c|c|c|c|}
\hline$\overline{V_{b}(\mathrm{~V})}$ & \multicolumn{2}{|c|}{1625} & \multicolumn{2}{|c|}{1500} & \multicolumn{2}{|c|}{1350} & \multicolumn{2}{|c|}{1000} & \multicolumn{2}{|c|}{1200} & \multicolumn{2}{|c|}{1000} & \multicolumn{2}{|c|}{262} \\
\hline$I_{b}(\mathrm{~mA})$ & \multicolumn{2}{|c|}{38} & \multicolumn{2}{|c|}{31.6} & \multicolumn{2}{|c|}{26.6} & \multicolumn{2}{|c|}{19.3} & \multicolumn{2}{|c|}{13.2} & \multicolumn{2}{|c|}{4.8} & \multicolumn{2}{|c|}{1.85} \\
\hline$P_{R F G}(\mathrm{~W})$ & & & & & & & & & & & & & & \\
\hline & Mod. & Exp. & Mod. & Exp. & Mod. & Exp. & Mod. & Exp. & Mod. & Exp. & Mod. & Exp. & Mod. & Exp. \\
\hline$\overline{T_{1}\left({ }^{\circ} \mathrm{C}\right)}$ & 111 & 113 & 108 & 108 & 104 & 104 & 82 & 88 & 66 & 76 & 49 & 57 & 46 & 54 \\
\hline$T_{2}\left({ }^{\circ} \mathrm{C}\right)$ & 120 & 120 & 117 & 115 & 112 & 111 & 90 & 92 & 70 & 79 & 51 & 58 & 48 & 55 \\
\hline$T_{3}\left({ }^{\circ} \mathrm{C}\right)$ & 90 & 93 & 88 & 89 & 85 & 86 & 68 & 73 & 57 & 64 & 42 & 49 & 42 & 48 \\
\hline$T_{4}\left({ }^{\circ} \mathrm{C}\right)$ & 86 & 86 & 84 & 83 & 82 & 80 & 66 & 66 & 55 & 58 & 41 & 45 & 41 & 44 \\
\hline$T_{5}\left({ }^{\circ} \mathrm{C}\right)$ & 112 & 117 & 110 & 113 & 108 & 110 & 88 & 92 & 72 & 79 & 53 & 59 & 50 & 55 \\
\hline$T_{6}\left({ }^{\circ} \mathrm{C}\right)$ & 142 & 151 & 140 & 147 & 135 & 144 & 112 & 122 & 90 & 104 & 66 & 77 & 62 & 72 \\
\hline$T_{7}\left({ }^{\circ} \mathrm{C}\right)$ & 53 & 58 & 53 & 56 & 51 & 55 & 42 & 47 & 37 & 43 & 31 & 36 & 31 & 36 \\
\hline$T_{8}\left({ }^{\circ} \mathrm{C}\right)$ & 63 & 58 & 63 & 55 & 60 & 55 & 49 & 47 & 42 & 43 & 34 & 36 & 34 & 36 \\
\hline
\end{tabular}

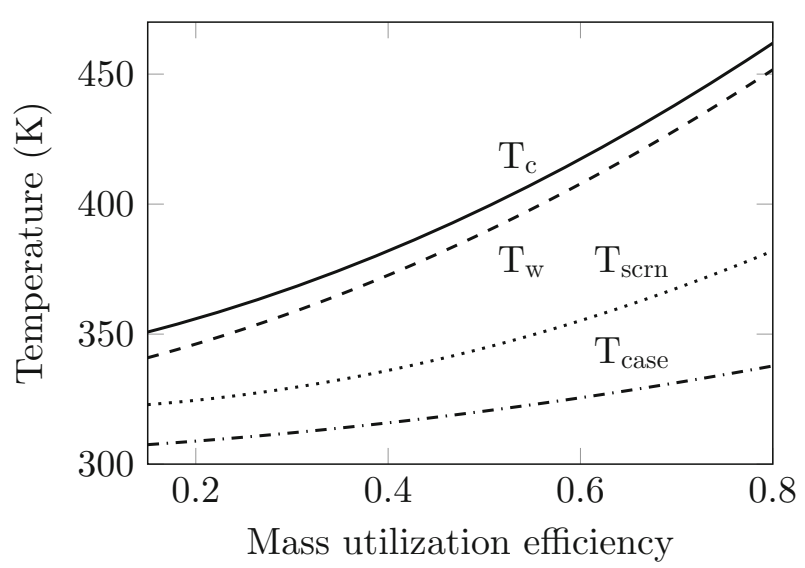

Fig. 13. Thruster's temperature distribution for different mass utilization efficiency values $\eta_{m}$ for a constant mass flow rate of $0.5 \mathrm{sccm}$

and case $T_{\text {case }}$ temperatures vary with the mass utilization efficiency. As the plot depicts, the coil and discharge chamber temperatures are very similar to each other with only a few percent difference. The screen grid temperature changes from being about $8 \%$ lower to $20 \%$ lower than the chamber wall temperature as the mass utilization efficiency increases. The case temperature is just above room temperature.
We continue our investigation by plotting the RFG input power variation with the mass utilization efficiency. This is done for three different cases. Case I represents a situation in which the thruster's temperatures are consistent with the plasma parameters and the RF heating, which is what occurs in the real-life situations. Case II represents a condition where the plasma parameters are consistent with the thruster's temperatures, but the RF heating is not. Finally, Case III simulates a situation in which the thruster's temperatures are set constant and not consistent with neither the plasma parameters nor the $\mathrm{RF}$ heating. Case III is commonly used in analyzing RF thrusters, where the discharge chamber wall temperature is set to a constant predefined value, usually around $400 \mathrm{~K}$. As can be seen in Figure 14, at low mass utilization efficiencies, Case III results in the RFG input power that is 3-4\% larger compared to other cases because it over estimates the thruster's temperatures. As we approach a mass utilization efficiency of around 0.5, we see that all three cases merge, since the thruster's temperatures are approximately equal to the constant temperatures that were set. However, as we approach high mass utilization efficiencies of 0.7 and 0.8 , the discrepancy between the cases increases. In particular, Case III under-estimates the power compared to Case I by up to $10 \%$, which is approximately a $2-3 \mathrm{~W}$ difference.

To finish off our discussion about the temperature effects on the plasma properties and thruster's performance, 


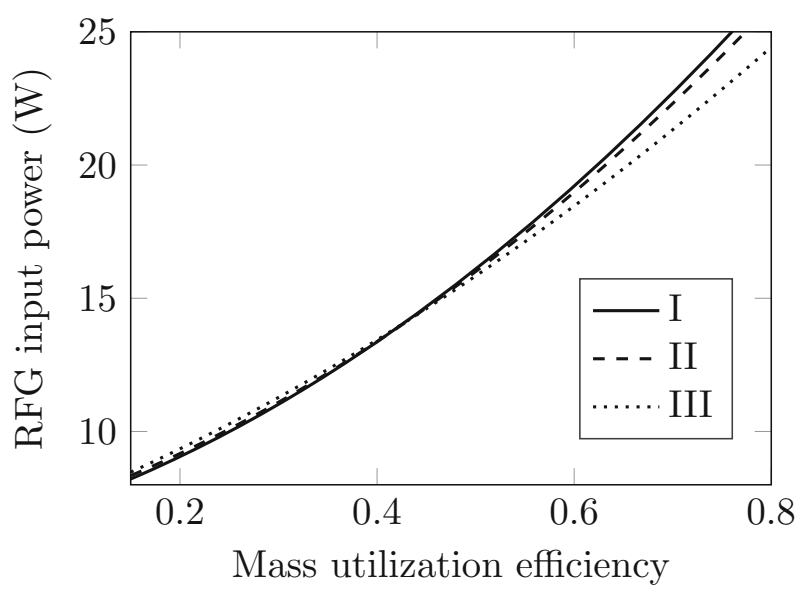

Fig. 14. RFG input power variation with the mass utilization efficiency for three difference thruster's temperatures-plasma properties-RF heating coupling cases.

we create the following hypothetical situation. First, we set the beam current $I_{b}$ that we are extracting to $20.3 \mathrm{~mA}$ and set the mass flow rate $\dot{m}_{i n}$ to 0.5 sccm as constants. Then we imagine that the thruster starts heating up, which could be due to a neutralizer starting to operate or due to a change in the thermal load from the spacecraft. This could also be because the thruster is exposed to different heat fluxes from the environment when, for instance, it starts facing the sun side. It could also simply mean a modification in the thruster's design causing the temperatures to change. The goal is to see how the thruster's temperatures affect the plasma parameters and the performance. The results of the analysis are plotted in Figure 15. All plots are done against the chamber wall temperature $T_{w}$, but it should be noted that all thruster's temperatures change.

First we see that as the temperatures increase, the neutral gas density $n_{0}$ decreases following an inversely proportional relationship $\left(n_{0} \propto 1 / T_{w}\right)$. In contrast, the electron temperature goes up in a linear fashion. Ion density, on the other hand, decreases linearly since an increase in the electron temperatures results in an increase in the Bohm velocity. Therefore, a lower ion density is needed to achieve a set beam current. The reflected thruster structural resistance $R_{\text {strct }}^{\prime}$, which is the thruster resistance excluding the plasma resistance, increases nearly linearly with the temperatures due to an increase in the thruster's component electrical conductivity. The reflected plasma resistance $R_{p}^{\prime}$ actually goes down since the plasma electrical conductivity decreases due to the decreasing ion density. Therefore, due to the combination of these effects, the total reflected thruster resistance $R_{t h r}^{\prime}$ goes up slightly. The coil current increases quite sharply because of two factors. First, due to an increasing electron temperature, there are higher boundary losses incurred, requiring more power (proportional to the coil current) to be transferred to the plasma. Second, due to the decrease in the plasma electrical conductivity, a higher coil current is required to transfer the same amount of power to the plasma. Finally, due to the increase in both the coil current and thruster resistance, the RFG input power goes up by about $20 \%$ when the wall temperature changes from 310 to $480 \mathrm{~K}$. Therefore, to increase the thruster's performance, it is beneficial to run the thruster at the lowest temperatures possible.

Figures 16 and 17 show the electromagnetic properties of the plasma. In Figure 16 we see that the maximum magnetic field inside the plasma is about 20 Gauss, whereas the majority of the plasma volume has a magnetic field of about 12 Gauss. Also in the majority of the plasma volume, the magnetic field is directed axially. In Figure 17 we see that most of the power is absorbed in the middle of the discharge due to end coil effects. Near the plasma center and axial discharge chamber edges, there is virtually no power absorption from the coil, which is indicated by the dark regions in the plots. The induced current density vectors are directed azimuthally and follow the power absorption trend with the highest current density present in the middle of the plasma, next to the coil.

\section{Conclusion}

A radio-frequency gridded-ion thruster performance and thermal model, based on the RIT 3.5 thruster geometry and experimental results, has been presented. The goal when designing the model was to make as few assumptions as possible, but at the same time have a model that represents real-life conditions, is accurate, produces easily interpretable results and has short solution times (less than an hour). It has been shown that the model can predict all the main RIT 3.5 thruster performance and thermal parameters with approximately $10 \%$ error. The model takes into account the plasma parameters, ion extraction, electromagnetic field distribution, RF circuit and temperature distribution. Therefore, for the first time, a model has been created that predicts the thruster's performance and temperature distribution in a self-consistent/coupled fashion. This is because the model includes the thruster's temperature effect on the plasma parameters/performance and vice versa. Such a coupling is representative of real-life conditions observed while testing RF thrusters. The developed model is in contrast to the other RF thruster models (developed for example by Volkmar [13,30], Chabert [12] or Gartner [14]) that only looked at a specific aspect of the $\mathrm{RF}$ thruster operation, concentrating on either predicting the thruster's performance or the temperature distribution but not both in a coupled fashion. It was observed that if the coupling between the thruster's temperatures and performance is not consistent, an error of up to about $10 \%$ in the RFG input power can be incurred at high mass utilization efficiencies. The error, however, depends on the thruster's temperatures that are set as a constant in performing the simulations. The $10 \%$ error was achieved when the chamber wall temperature was set to $400 \mathrm{~K}$, which is a common value used when analyzing the $\mathrm{RF}$ thruster performance.

In particular, the model was also used to observe the temperature effect on the thruster's performance. It was noticed that as thruster's temperatures increase, the coil current and reflected thruster resistance also increase. 


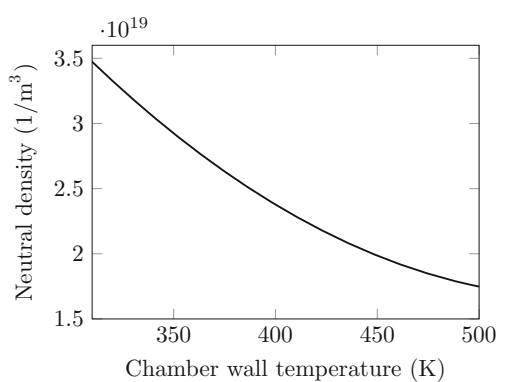

(a)

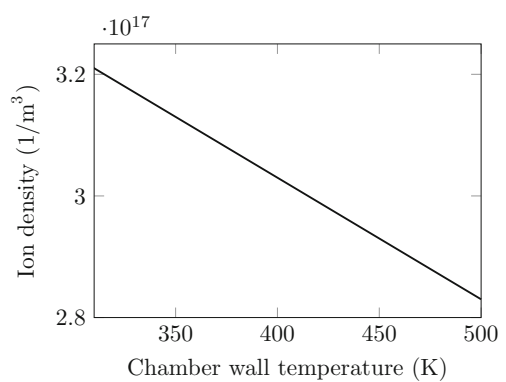

(c)

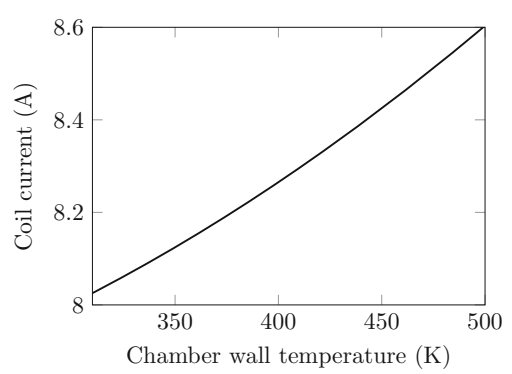

(e)

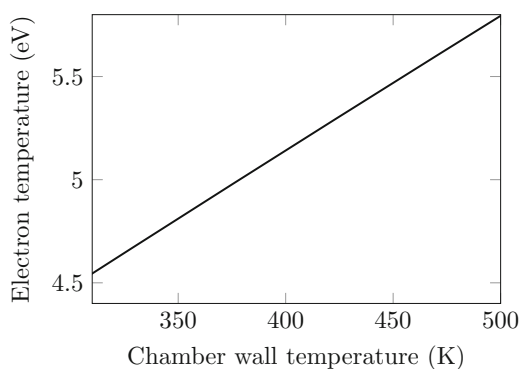

(b)

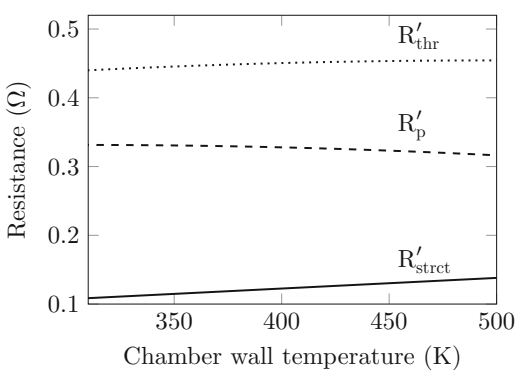

(d)

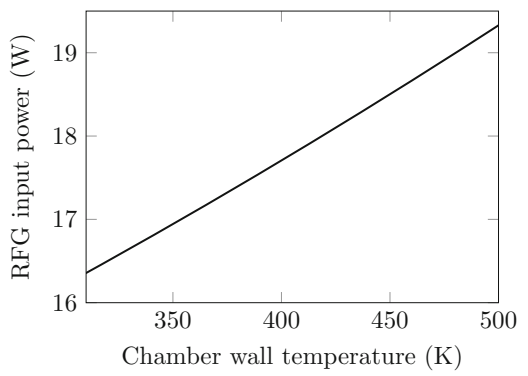

(f)

Fig. 15. (a)-(c) Plasma properties, (d) thruster resistance, (e) coil current and (f) RFG input power as functions of the chamber wall temperature for a constant mass flow rate of $0.5 \mathrm{sccm}$ and a beam current of $20.3 \mathrm{~mA}$.

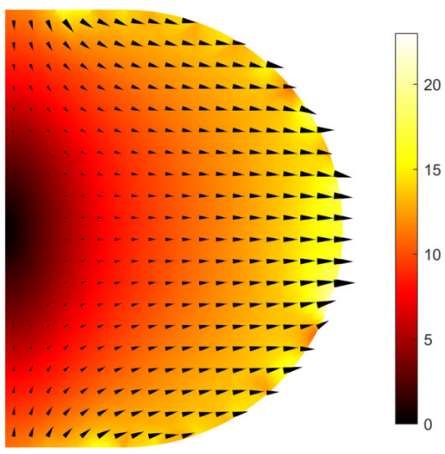

Fig. 16. Magnetic field strength in Gauss and magnetic flux density vectorial direction inside the plasma.

This results in the RFG input power going up. For instance, it was observed that if the thruster's chamber wall temperature rises from 310 to $480 \mathrm{~K}$, the RFG input power goes up by about $20 \%$. This shows that it is important to keep the thruster as cool as possible. This could be

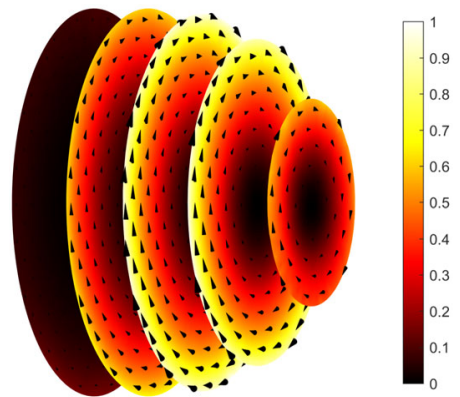

Fig. 17. Normalized power absorption magnitude and vectorial induced current density distribution inside the plasma.

achieved by changing the thruster's design. Additionally, when planning a space mission, the environmental thermal fluxes to the thruster have to be minimized. This is especially true for missions that work with low power thrusters. The model can also be used to judge the thermal thruster design because it provides a 3D surface temperature distribution plot. By investigating the RIT 3.5 
temperature distribution plot, it was observed that the coil temperature gets above $200{ }^{\circ} \mathrm{C}$, which makes it the hottest component in the thruster. Considering that the copper which is commonly used to manufacture coils has quite a high thermal expansion coefficient, a change in the coil's shape could reduce the thruster's performance. What is more, the matching capacitors reach about $150{ }^{\circ} \mathrm{C}$. The designer must check that the capacitors' temperatures do not go above around $200{ }^{\circ} \mathrm{C}$ throughout the operational envelope of the thruster. This can now be achieved using the model.

The model can potentially be used to gauge different thruster geometries and performances, and act as a tool for designing and optimizing $\mathrm{RF}$ ion thrusters to make them more attractive for specific space missions. The future goal is to introduce a possibility of including environmental heat fluxes based on a specific mission orbit. It is also possible to add to the model a possibility of accounting for mechanical deformations of the coil and grids due to thermal stresses, and to check how these affect the thruster's performance.

The authors would like to thank Johann Müller for his useful comments regarding the RFG physics and general RF thruster working principles. This research was funded by the Engineering and Physical Sciences Research Council (EPSRC): funding reference - 1362213 .

\section{References}

1. W. Hittorf, Ann. Phys. Chem. 21, 90 (1884)

2. H.W. Loeb, J. Spacecraft Rockets 8, 494 (1970)

3. R. Killinger, H. Leiter, R. Kukies, in the $43 r d$ AIAA/ASME/SAE/ASEE Joint Propulsion Conference $\mathcal{E}$ Exhibit, Cincinnati, OH, 2007

4. M. Kilter, A. Karlsson, in Proceedings of the 4 th International Spacecraft Propulsion Conference, Sardinia, Italy, 2004

5. H. Leiter et al., in the $4^{\text {rth }}$ AIAA/ASME/SAE/ASEE Joint Propulsion Conference and Exhibit, San Diego, CA, 2011

6. D. Feili et al., in the 30th ISTS, 34th IEPC, 6th NSAT Joint Conference, Kobe-Hyogo, 2015

7. J.J. Thompson, Phil. Mag. 32, 321 (1890)

8. R.B. Piejak, V. Godyak, B.M. Alexandrovich, Plasma Sources Sci. Technol. 1, 179 (1992)

9. J.T. Gudmundsson, M.A. Lieberman, Plasma Sources Sci. Technol. 6, 540 (1997)
10. G.G. Lister, Y.M. Li, V.A. Godyak, J. Appl. Phys. 79, 8993 (1996)

11. D.M. Goebel, IEEE Trans. Plasma Sci. 36, 2111 (2008)

12. P. Chabert, J. Arancibia Monreal, J. Bredin, L. Popelier, A. Aanesland, Phys. Plasmas 19, 073512 (2012)

13. C. Volkmar, U. Ricklefs, Eur. Phys. J. D 69, 227 (2015)

14. W. Gartner, B. Lotz, B.K. Meyer, in the 33rd International Electric Propulsion Conference, Washington, D.C, 2013

15. J. Van Noord, in the 43rd Joint Propulsion Conference and Exhibit, Cincinnati, OH, 2007

16. COMSOL Group, "COMSOL", multi-Physics Software Package, Ver. 5.1, Stockholm, 2015

17. P. Chabert, N. Braithwaite, Physics of Radio-Frequency Plasmas (Cambridge University Press, Cambridge, 2011)

18. D. Goebel, I. Katz, Fundamentals of Electric Propulsion (John Wiley \& Sons, Hoboken, NJ, 2005)

19. A. Lieberman, M. Lichtenberg, Principles of Plasma Discharges and Materials Processing, 2nd edn. (John Wiley \& Sons, New Jersey, 2005)

20. T. Kalvas, O. Tarvainen, T. Ropponen, O. Steczkiewicz, J. Arje, H. Clark, Rev. Sci. Instrum. 81, 02 B703 (2010)

21. Cadence Design Systems Inc., "PSPICE", electronics Circuit Modelling Software Package, Ver. 9.1, San Jose, CA, 2015

22. J. Meunier, Ph. Belenguer, J.P. Boeuf, J. Appl. Phys. 78, 731 (1995)

23. Y.M. Aliev, A.V. Bychenkov, H. Schluter, Philos. Mag. 4, 2413 (1967)

24. V.A. Godyak, R.B. Piejak, B.M. Alexandrovich, Phys. Rev. Lett. 80, 3264 (1998)

25. G. Hagelaar, L. Pitchford, Plasma Sources Science and Technology, 14, 722 (2005)

26. M. Schäfer, Ph.D. Dissertation, Physics Dept., University of Giessen, Giessen, 1971

27. I. Shkarofsky, T. Johnston, M. Bachynski, The Particle Kinetics of the Plasmas (Addison-Wesley, 1996)

28. J. Yogesh, E.T. Kenneth, Computational Heat Transfer, 1st edn. (Hemisphere Publishing Corporation, SpringerVerlag, Berlin, 1986)

29. A. Bejan, A.D. Kraus, Heat Transfer Handbook, 1st edn. (John Wiley \& Sons, Hoboken, New Jersey, 2003)

30. C. Volkmar, U. Ricklefs, in the 2014 Space Propulsion Conference, Cologne, 2014

Open Access This is an open access article distributed under the terms of the Creative Commons Attribution License (http://creativecommons.org/licenses/by/4.0), which permits unrestricted use, distribution, and reproduction in any medium, provided the original work is properly cited. 